\title{
Glutamate et mGluR1: la clé et la serrure de l'apprentissage chez les mammifères
}

Il est maintenant généralement admis que le glutamate est l'acide aminé excitateur responsable de l'activation de certains neurones impliqués dans les processus d'apprentissage et de mémorisation du système nerveux central. On a pu identifier in vitro deux familles de récepteurs du glutamate : l'une est constituée de récepteurs ioniques (iGluRs) activés par le NMDA et qui permettent l'entrée dans la cellule d'ions calcium. L'autre famille est constituée de récepteurs à 7 segments transmembranaires (7TMD) qui, parce qu'ils sont couplés à différentes protéines $G$, transmettent le signal à un certain nombre d'effecteurs intracellulaires. Ces récepteurs à 7TMD sont appelés récepteurs métabotropiques ou mGluR [1]. De nombreuses études in vitro ont montré, à l'aide d'antagonistes, l'implication de ces deux familles de récepteurs dans des processus de renforcement de l'activité synaptique tels que la potentialisation à long terme (LTP) dans l'hippocampe et la dépression à long terme (LTD) dans le cortex cérébelleux. Si la présence des récepteurs sensibles au NMDA est nécessaire pour l'induction de la LTP dans certaines connexions de l'hippocampe, elle n'est pas indispensable car il existe des zones où la LTP peut être induite en l'absence de ces récepteurs. En revanche, il a été récemment montré qu'aucune forme de renforcement synaptique à long terme ne peut être produite sans l'activation des $\mathrm{mGluR}$, et cela dans l'hippocampe comme dans le cervelet [2]. Les études sur les mGluR se sont heurtées à divers obstacles dont l'absence d'antagonistes efficaces et sélectifs pour les diffé$m / s n^{\circ} 2$, vol. 11, férier 95 rents sous-types de récepteurs (mGluR1-7) qui composent cette famille. Afin de résoudre ces problèmes, nous avons produit, par recombinaison homologue, une lignée de souris mutantes pour le gène codant pour mGluRl [3].

Cette technique consiste à inactiver le gène cible dans des cellules souches embryonnaires (ES), par intégration d'un vecteur de recombinaison homologue dans le locus du gène. Les cellules ES sont ensuite injectées dans un embryon hôte et, grâce à leur capacité de coloniser tous les tissus de la souris y compris la lignée germinale, la mutation est alors transmise à la descendance. Pour ce qui concerne mGluRl, nous en avons inactivé le gène dans les cellules ES tout en plaçant en phase le gène marqueur lac $Z$, afin d'établir une corrélation entre d'éventuels phénotypes et l'absence de mGluRl dans certains tissus, via l'expression de la $\beta$-galactosidase ( $\beta \mathrm{Gal})[4]$.

La mutation de mGluRl a provoqué chez l'animal un déficit moteur sévère qui s'apparente à l'ataxie. Ce phénotype apparaît de manière soudaine deux semaines après la naissance. Une analyse complète de l'activité motrice révèle que la tension musculaire ainsi que le poids de l'animal sont normaux. L'origine de ce déficit moteur est très probablement cérébelleuse. L'histologie du cerveau n'a pourtant rien révélé d'anormal : aucune distorsion dans les diverses couches cellulaires ni absence de cellules dans le cortex cérébelleux ou dans l'hippocampe n'ont été remarquées. En outre, nous avons montré, à l'aide d'anticorps spécifiques, que le récepteur était non seulement absent mais aussi qu'il avait été bel et bien remplacé par la protéine de fusion $\mathrm{mGluRl} / \beta \mathrm{Gal}$ (figure 1). De plus, nous avons pu identifier et analyser plus précisément les tissus qui normalement expriment mGluRl, à l'aide de l'expression $\beta \mathrm{Gal}$. Là encore, aucune différence anatomique n'a pu être détectée entre une souris de type sauvage et une souris mutante homozygote.

Comme de nombreuses études ont montré que mGluRl était fortement exprimé dans les cellules de Purkinje, l'analyse électrophysiologique du cortex cérébelleux a été effectuée, principalement au niveau des connexions entre les cellules de Purkinje et les fibres parallèles et grimpantes. Une fois encore, aucune anomalie n'a pu être détectée concernant l'amplitude de différents types de courants calciques ou sodiques. De plus, nous avons pu montrer qu'un agoniste des mGluR, l'ACPD (aminocyclopentane-1,3dicarboxylate), n'avait plus d'effet sur les cellules de Purkinje mutantes, confirmant ainsi l'absence fonctionnelle des récepteurs mGluRl. Ces résultats suggèrent que les récepteurs mGluRl ne participent donc pas à l'activité électrochimique de base des synapses dans le cortex cérébelleux. Par ailleurs, puisqu'il a été proposé que les mGluR étaient impliqués dans les processus qui président à l'apprentissage moteur, la LTD a été analysée sur tranches de cervelet maintenues artificiellement en vie. Le protocole consiste à coupler une stimulation de la fibre parallèle à une dépolarisation de la cellule de Purkinje. Une dépression persistante du potentiel synaptique apparaît normalement au niveau de la synapse entre les deux cellules. 


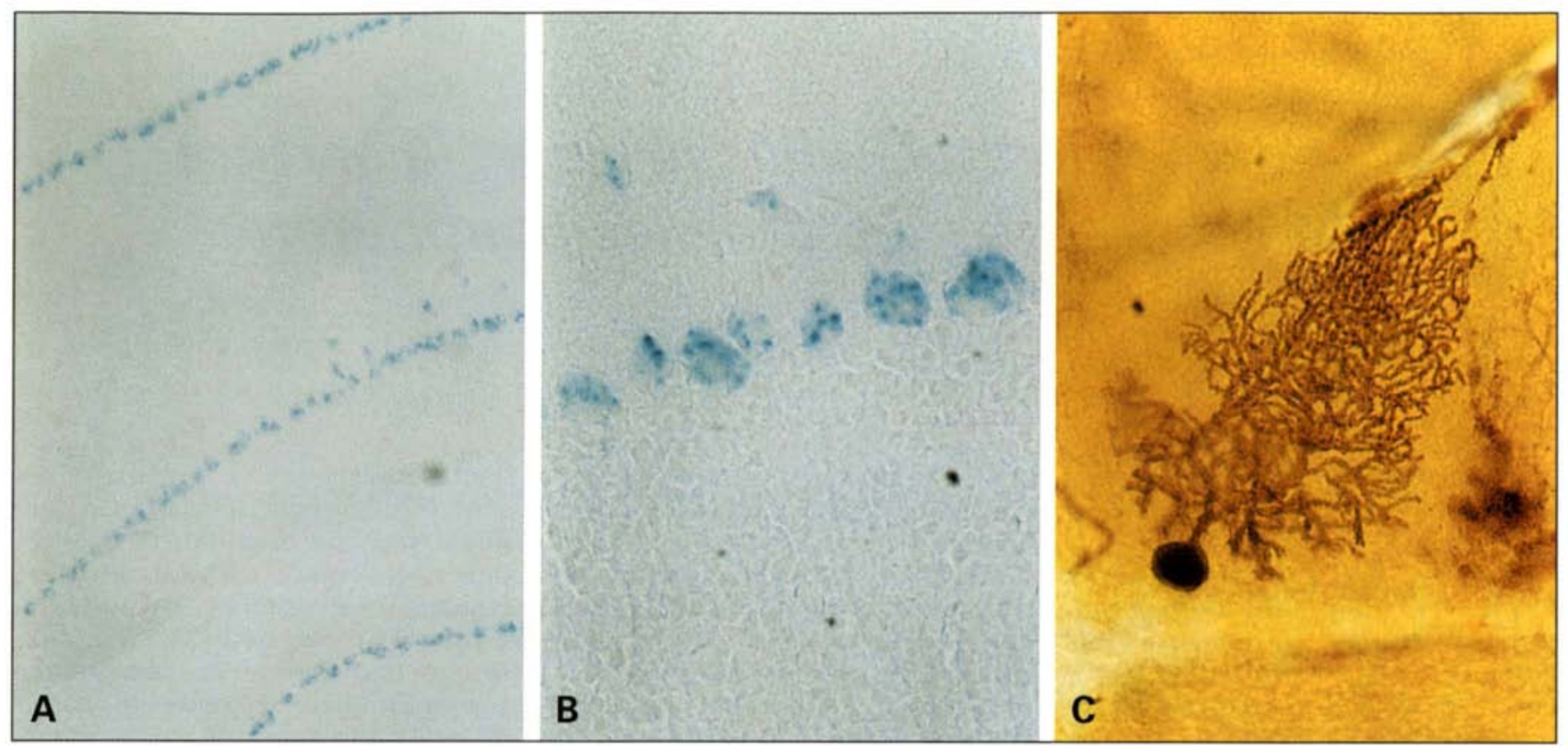

Figure 1. A et B. Mise en évidence du remplacement du gène de mGluR1 par la $\beta$-galactosidase. L'expression de mGluR1 étant relativement forte dans les cellules de Purkinje, le test de coloration au X-Gal fait apparaître le corps cellulaire coloré en bleu de manière spécifique sur cette seule couche de cellules, respectivement à faible et fort grossissement. C : Morphologie inchangée d'une cellule de Purkinje de souris mutante, colorée à la peroxydase.

Après analyse complète des deux types de souris, il ressort de cette étude qu'aucune LTD significative n'a pu être détectée dans le cervelet des souris mutantes, alors qu'une dépression forte et persistante a pu être induite chez les souris de type sauvage. Ce résultat nous amène donc à conclure que les récepteurs mGluRl sont nécessaires à l'induction de la LTD dans le cervelet et qu'ils jouent un rôle essentiel dans la coordination motrice chez l'animal. Il est toutefois prématuré de conclure que l'absence de LTD est responsable du déficit moteur chez les animaux mutants.

Puisqu'il a été suggéré, à l'issue d'études in vitro, que certains soustypes de mGluR pouvaient jouer un rôle dans les processus d'apprentissage et de mémorisation, nous avons soumis les souris mutantes mGluRl-/au test comportemental de Morris. Il capacités de mémorisation spatiale pour se sortir d'une situation dangereuse. On sait que ce type de mémorisation requiert l'activité de l'hippocampe. Ce test se décompose en deux parties afin de ne mettre en évidence que l'élément responsable de la mémorisation spatiale. Lors du premier exercice, les animaux sont placés dans un bassin rempli d'eau rendue trouble avec du lait. Quelque part dans le bassin se trouve une plate-forme, placée légèrement en dessous du niveau de l'eau et rendue repérable par la souris à l'aide d'un drapeau. Les animaux sont placés à divers endroits du bassin et apprennent rapidement à trouver la plateforme en se dirigeant vers le drapeau. Les souris témoins ainsi que les souris mutantes ont été entraînées de la sorte et il est apparu qu'à l'issue de trois séances, les deux groupes de souris avaient les mêmes capacités visuelles associant le drapeau à la plate- forme. En outre, on peut constater que les souris mutantes, bien qu'ataxiques sur support solide, se comportent normalement dans l'eau. Dans le deuxième exercice, les animaux sont entraînés de la même manière mais, cette fois-ci, le drapeau a été retiré de la plate-forme. De plus, on place sur les murs de la pièce des repères visuels. Il n'y a donc plus d'association directe entre la plateforme et un indice visuel. La souris doit faire un effort d'abstraction afin d'imaginer la localisation de la plateforme en fonction d'indices indirects. Cet apprentissage spatial est directement imputable à l'activité de mémorisation de l'hippocampe. Au cours de ce deuxième exercice, les souris mutantes mGluRl-/- n'ont jamais été capables de localiser la plate-forme, alors que les souris témoins, comme pour le premier exercice, apprirent rapidement cette tâche. On peut conclure de ces deux 


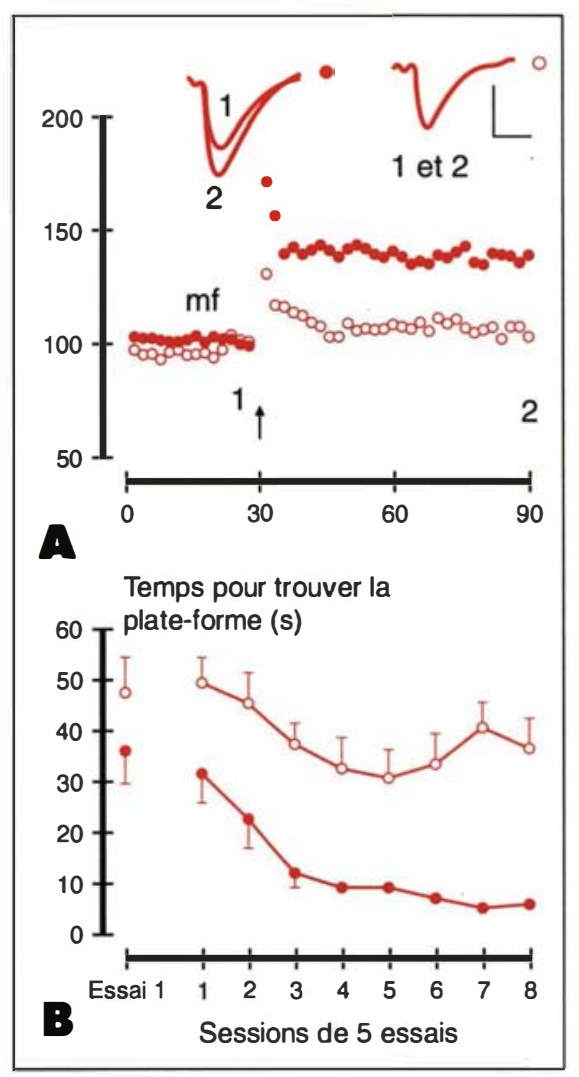

Figure 2. A. Absence de LTP dans les fibres moussues (mf) chez les souris mutantes (cercles vides), et des souris de type sauvage (cercles rouges). Représentation du pourcentage de mfLTP en fonction du temps (min). L'amplitude de la mfLTP est considérée comme $100 \%$ avant stimulation représentée par la flèche $1100 \mathrm{~Hz}$, $1 \mathrm{~s})$. Les barres de calibration sont: $0,25 \mathrm{mV}$ en ordonnée et $5 \mathrm{~ms}$ en abscisse. La superposition des courbes 1 et 2 représente l'amplitude relative de la potentiation, respectivement avant et après stimulation; on observe une différence significative entre 1 et 2 chez les souris de type sauvage alors qu'aucune différence n'est visible chez les souris mutantes. B. Test d'apprentissage représentant la capacité des souris mutantes (cercles vides) et témoins (cercles pleins) à trouver la plate-forme. Aucune amélioration dans la performance n'a été détectée chez les souris mutantes.

$m / s n^{\circ} 2$, vol. 11, févirier 95

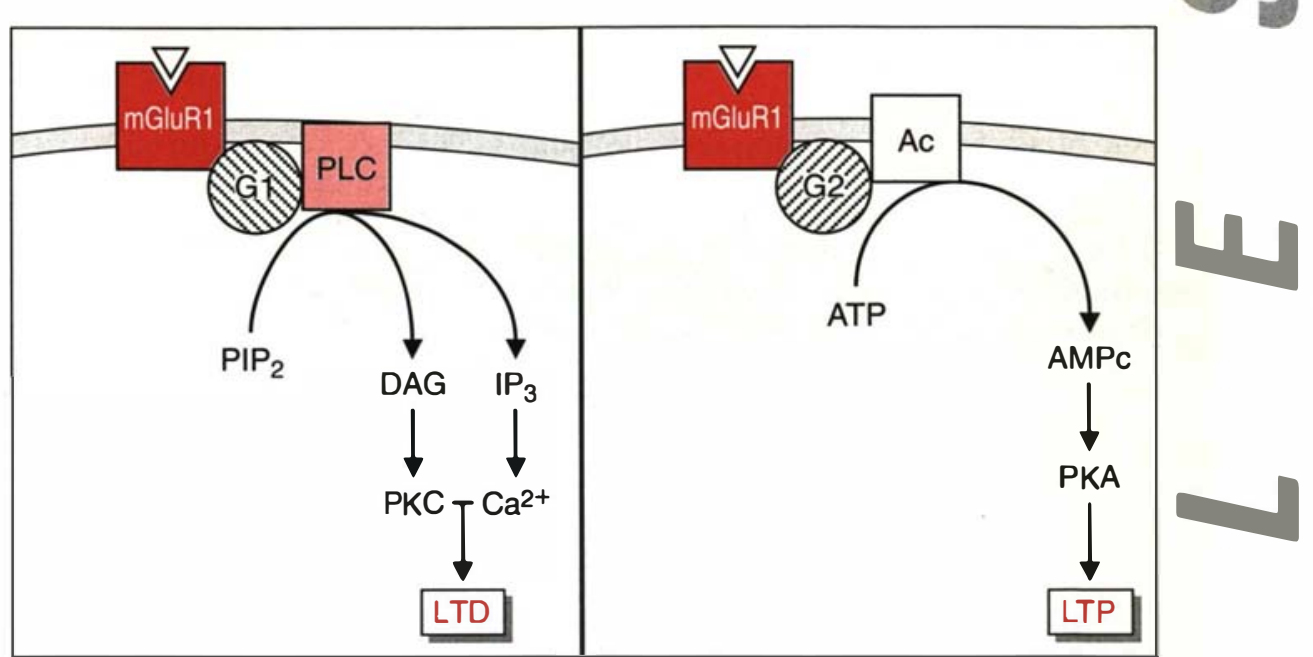

Figure 3. Représentation schématique des mécanismes d'action induisant la LTD dans le cervelet (A) et la LTP dans les fibres moussues de l'hippocampe (B). Les couplages se font avec deux protéines G différentes (G1 et G2) selon le tissu. Le second messager est, soit le couple diacylglycérol /inositol trisphosphate (DAG / IP3), issu de l'activation de la phospholipase C (PLC), soit I'AMPc, issu de l'activation de l'adénylyl cyclase (Ac). La deuxième partie de la cascade d'activation met en œuvre deux protéine kinases : $C$ (PKC) pour la LTD et A (PKA) pour la mfLTP. PIP2 : phosphatidyl inositol bisphosphate.

tests que les souris mutantes n'ont pas de déficits visuels et sont capables d'associer un indice direct avec l'objectif final. En revanche, elles sont incapables d'intégrer et de mémoriser de nouvelles informations qui requièrent un effort d'abstraction.

Pour compléter cette étude, il était logique d'analyser la LTP de ces souris mutantes afin d'établir s'il existe une corrélation entre l'apprentissage spatial et ce modèle électrophysiologique de mémorisation dans l'hippocampe $\left(m / s n^{\circ} 1\right.$, vol. 7, p. 80$)$. Ainsi, après avoir vérifié que les différentes connexions synaptiques des trois faisceaux majeurs de l'hippocampe étaient intactes et fonctionnelles, nous avons procédé à l'étude de la LTP dans cinq zones différentes de l'hippocampe. Il ressort de cette étude que seule la LTP analysée au niveau des fibres moussues de la région CA3 (mfLTP) n'a pu être induite chez les souris mutantes. Toutes les autres formes de LTP connues, en particulier celle habituellement étudiée dans la région CAl de l'hippocampe, sont intactes. On peut donc établir un lien entre l'absence de mfLTP et l'incapacité de mémorisation spatiale des souris mutantes mGluRI-/- (figure 2).

En conclusion, il peut sembler surprenant que l'absence d'un seul récepteur soit à l'origine d'un tel déficit moteur sans qu'il y ait la moindre anomalie anatomique chez l'animal analysé dans son ensemble. Ce phénotype étant probablement d'origine cérébelleuse et mGluRl étant fortement exprimé dans les cellules de Purkinje, il est donc raisonnable d'envisager que les récepteurs mGluRl représentent un facteur clé dans le rôle régulateur de la fonction motrice exercée par les cellules de Purkinje au sein du cortex cérébelleux. De plus, à ces fonctions modulatrices, on peut ajouter à présent, au niveau
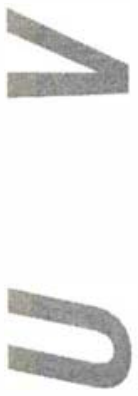
synaptique, la dépression à long terme. Cela nous a permis de valider pour la première fois, au niveau physiologique, le modèle que constitue la LTD dans le cervelet.

Par ailleurs, l'étude comportementale de ces souris mutantes a définitivement apporté la preuve que les mGluR en général et mGluRl en particulier étaient impliqués dans les processus de mémorisation et d'apprentissage. A ce propos, il a été récemment montré, à l'aide d'antagonistes, que mGluR2 était responsable de la mémoire olfactive chez le rat. Le fait que la mfLTP soit absente chez les souris mutantes apporte un élément supplémentaire d'information concernant la région de l'hippocampe responsable de l'apprentissage spatial. Il est à noter, en outre, que les deux types de renforcement synaptique que sont la LTD dans le cervelet et la mfLTP dans l'hippocampe sont induits selon un mécanisme indépendant de l'activation des récepteurs sensibles au NMDA, alors que les autres types de LTP impliquent l'activation de ces mêmes récepteurs (figure 3). Cela pourrait suggérer un processus général d'association avec les récepteurs sensibles au NMDA impliquant un autre sous-type de mGluR tel que mGluR5. Cette hypothèse sur le rôle de mGluRl dans la plasticité synaptique nous amène donc à penser que ce récepteur serait phylogénétiquement plus primitif que mGluR5 qui ne fonctionnerait, quant à lui, que selon un mode associatif. MGluRl serait alors le récepteur res- ponsable de la plasticité homosynaptique, que l'on a décrit comme étant responsable du processus d'apprentissage chez d'autres espèces animales telles que la mouche ou certains mollusques.

F.C.

1. Nakanishi S. Molecular diversity of glutamate receptors and implications for brain function. Science 1992 ; 258 : 597-603.

2. Bliss TVP, Collingridge GL. A synaptic model of memory: long-term potentiation in the hippocampus. Nature $1993 ; 361: 31-9$.

3. Conquet F, Bashir Zl, Davies $\mathrm{CH}$, et al Motor deficit and impairment of synaptic plasticity in mice lacking mGluRl. Nature $1994 ; 372$ : 237-43. 4. Babinet C. Les cellules souches embryonnaires de souris : une voie privilégiée de transformation génétique à l'échelle de l'animal. médecine/ sciences $1992 ; 8$ : 268-75.

\section{BRÈVES}

口 Polyadénylation du messager: de la drosophile à l'homme. Nous sommes loin de connaître toutes les étapes nécessaires et tous les facteurs impliqués dans la maturation des précurseurs des ARN messagers. Dans un article paru récemment dans Nature, Takagaki et Manley (New York, USA) identifient différents partenaires protéiques nécessaires au clivage du prémessager suivi de sa polyadénylation. L'un d'entre eux est le cleavage stimulating factor (CstF), composé de trois sousunités de 50, 64 et $77 \mathrm{kDa}$. L'ADNc de la sous-unité $77 \mathrm{kDa}$ humaine, dont le rôle est d'établir un pont entre les deux autres sous-unités, a été cloné. L'analyse de sa séquence montre que cette sous-unité est homologue de la protéine codée par le gène suppressor of forked $(S u(f))$ chez Drosophila melanogaster. Su(f) est un exemple de gènes dits modificateurs. Ces dernières années, chez Drosophila melanogaster, l'identification de tels gènes impliqués dans les fonctions de base de la cellule a été menée en étudiant les conséquences de leur mutation sur l'expression de gènes rapporteurs indépendants. L'approche a été la suivante : il existe de nombreux allèles hypomorphes dus à l'insertion d'éléments transposables qui apportent avec eux de nouveaux signaux d'épissage ou de polyadénylation reconnus par la machinerie cellulaire de l'hôte. Si une mutation touche l'un des gènes modificateurs sous le contrôle desquels s'est établie l'expression du gène muté par l'élément transposable, alors l'expression de ce dernier est modifiée et, par voie de conséquence, le phénotype de l'individu. Ainsi, en contexte Su(f) sauvage, le gène forked, muté par l'insertion d'un élément transposable nommé Gypsy, confère à la drosophile des soies fourchues. Ce phénotype mutant disparaît en contexte $S u(f)$ muté. Avec la mise en évidence du rôle joué par la protéine codée par $S u(f)$, Takagaki et Manley montrent que, au-delà du phénomène de transcription, les différents événements conduisant à la maturation de l'ARN, tels que la polyadénylation ou l'épissage, jouent un rôle important dans la régulation de l'ex- pression génique. Ce travail suscite plusieurs remarques : (1) les mécanismes conduisant à la maturation des messagers sont très conservés de la drosophile à l'homme; (2) l'isolement des facteurs et des gènes contrôlant de telles fonctions cellulaires pourrait, par conséquent, permettre de comprendre les mécanismes moléculaires mis en jeu lors de la dérégulation d'un gène produite par l'insertion de novo d'un élément transposable chez l'homme, situation dont on sait aujourd'hui qu'elle se rencontre dans certaines maladies humaines $\left(\mathrm{m} / \mathrm{s} n^{\circ} 2\right.$, vol. 8 , p. 169); (3) à terme, cela pourrait aboutir à fournir des outils pour comprendre des situations pathologiques jusque-là inexplorées ; (4) la connaissance du rôle des produits de gènes modificateurs identifiés chez la drosophile pourrait être à l'origine d'un chapitre nouveau de "modulation génétique à visée thérapeutique ".

[Takagaki Y, Manley JL. Nature 1994 ; 372 : 471-4.] 